\title{
Reviewers in this Issue
}

Dr. Hasan Md. Abdur Rouf

Professor of Surgery

Delta Medical College, Dhaka, Bangladesh

Dr. Shah Md. Zahurul Haque Asna

Professor of Microbiology

Bangladesh University of Health Sciences, Dhaka, Bangladesh

Dr. S. M. Fazlul Karim

Professor of Biochemistry

Delta Medical College, Dhaka, Bangladesh

Dr. Md. Nur Hossain

Professor of Forensic Medicine

Delta Medical College, Dhaka, Bangladesh

Dr. M S Zaman Shaheen

Professor of Orthopaedics

Dhaka Medical College, Dhaka, Bangladesh

Dr. Md. Rezwanur Rahman

Professor of Biochemistry

Delta Medical College, Dhaka, Bangladesh

Dr. Rehnuma Jahan

Consultant of Obs. \& Gynae

Square Hospital, Dhaka, Bangladesh

Dr. Samiron Kumar Mondal

Associate Professor of Surgery

BIRDEM Hospital \& Ibrahim Medical College, Dhaka, Bangladesh 